\title{
Lagrangian Gauge Structure Functions for Systems with First-Class Constraints
}

\author{
Domingo J. Louis-Martinez \\ Science One Program and \\ Department of Physics and Astronomy, \\ University of British Columbia \\ Vancouver, Canada
}

\begin{abstract}
The structure functions of the lagrangian gauge algebra are given explicitly in terms of the hamiltonian constraints and the first-order hamiltonian structure functions and their derivatives.
\end{abstract}


The importance of gauge symmetries in all modern relativistic theories of the fundamental interactions is well understood at present. Electromagnetic and Yang-Mills theories are examples of systems with natural bases of gauge generators that form closed lagrangian gauge algebras. The hamiltonian first-class constraints in these theories are linear in the canonical momenta. Their first-order hamiltonian gauge structure functions are constants and therefore the first-class constraints form Lie groups. In Einstein's theory of gravity one of the hamiltonian constraints is quadratic in the momenta [1] and the first-order hamiltonian gauge structure functions do not depend on the canonical momenta but do depend on the metric [2]. The hamiltonian constraints in Einstein's theory do not form a Lie group [2], but the natural basis of lagrangian gauge generators does form a closed gauge algebra.

The study of open lagrangian gauge algebras started with the discovery of supergravity $[3,4,5]$. In theories with open lagrangian gauge algebras we have to deal with lagrangian structure functions of higher orders. The existence of these structure functions has been established using an axiomatic approach in [6]. In the quantum theory, in order to construct the Feynman diagrams one needs first to determine all the gauge structure functions. Although these functions have been found explicitly in particular cases [4], their general form for the generic case of a hamiltonian system with first-class constraints is not known. The purpose of this paper is to solve this problem. We will present explicit expressions for the lagrangian gauge structure functions up to fourth order. We will show how these structure tensors are determined by the hamiltonian constraints and the hamiltonian firstorder structure functions. It is remarkable that to determine the higher order lagrangian gauge structure tensors no knowledge of the higher-order hamiltonian structure functions is required. They depend only on the zeroth- and first-order hamiltonian structure functions. The method presented here can be used to find the structure functions of even higher levels.

Let us consider a physical system described by the action:

$$
S=\int d t L
$$

$L$ is the Lagrangian of the system, which is a function defined in the velocity phase space $T Q$ (TQ is the tangent bundle of the n-dimensional configuration space $Q$ ). The variables $q^{i}(i=1,2, \ldots, n)$ are the generalized coordinates and $\dot{q}^{i}$ the generalized velocities.

The Euler-Lagrange equations of motion may be written in the following form:

$$
L_{i} \equiv W_{i j} \ddot{q}^{j}-\alpha_{i}=0
$$

where,

$$
\begin{gathered}
W_{i j} \equiv \frac{\partial^{2} L}{\partial \dot{q}^{i} \partial \dot{q}^{j}} \\
\alpha_{i} \equiv \frac{\partial L}{\partial q^{i}}-\dot{q}^{l} \frac{\partial^{2} L}{\partial q^{l} \partial \dot{q}^{i}}
\end{gathered}
$$

We assume that the dimension of the kernel of the Hessian matrix $W$ is constant (the same in all points of $T Q$ ) and equal to $m$ : 


$$
\operatorname{rank}\|W\|=n-m
$$

Let us denote as $R_{\mu}(q, \dot{q})(\mu=1,2, \ldots, m)$ a set of linearly independent null eigenvectors of the Hessian matrix:

$$
\begin{gathered}
R_{\mu}^{i} W_{i j} \equiv 0 \\
\operatorname{rank}\left\|R_{\mu}^{i}\right\|=m
\end{gathered}
$$

Using the Euler-Lagrange equations (2) and the identities (6) we obtain the lagrangian constraints of the first-level [7]:

$$
\chi_{\mu}^{(1)} \equiv R_{\mu}^{i} \alpha_{i}=0
$$

Let us assume that (8) do not bring about any restrictions in the velocity phase space $T Q$ (we assume that (8) are identities in $T Q$ ):

$$
R_{\mu}^{i} \alpha_{i} \equiv 0
$$

In this case, the left-hand-sides $L_{i}$ of the Euler-Lagrange equations (2) satisfy the Noether identities [7]:

$$
R_{\mu}^{i}(q, \dot{q}) L_{i}(q, \dot{q}, \ddot{q}) \equiv 0
$$

The Noether identities are satisfied by any trajectory $q(t)$ in the configuration space Q. According to the second Noether theorem [8], the action (1) is invariant under the infinitesimal gauge transformations:

$$
\delta q^{i}(t)=\epsilon^{\mu}(t) R_{\mu}^{i}(q(t), \dot{q}(t))
$$

$R_{\mu}^{i}$ are the generators of the gauge transformations (11).

It is not difficult to prove that under infinitesimal gauge transformations of the form (11) the left-hand-sides of the Euler-Lagrange equations (2) transform as follows:

$$
\delta L_{i}=\epsilon^{\mu}\left[-\frac{\partial R_{\mu}^{j}}{\partial q^{i}} L_{j}+\frac{d}{d t}\left(\frac{\partial R_{\mu}^{j}}{\partial \dot{q}^{i}} L_{j}\right)\right]+\dot{\epsilon}^{\mu} \frac{\partial R_{\mu}^{j}}{\partial \dot{q}^{i}} L_{j}
$$

From (7) it follows that we are dealing with an irreducible gauge theory $[5,9]$. The most general regular solution of the equation:

$$
\lambda^{i} L_{i} \equiv 0
$$

can be written as [6]:

$$
\lambda^{i}=R_{\mu}^{i} \epsilon^{\mu}+T^{i j} L_{j}
$$

where, 


$$
T^{i j}=-T^{j i}
$$

The trivial gauge transformations $T^{i j} L_{j}$ have no physical significance. However, in relativistic field theories trivial gauge transformations may appear as a result of requiring manifest covariance and preservation of locality.

Let us consider the commutator of two infinitesimal gauge transformations:

$$
\delta_{1} \delta_{2} q^{i}-\delta_{2} \delta_{1} q^{i} \equiv\left[\delta_{1}, \delta_{2}\right] q^{i}
$$

In general, the commutator of two infinitesimal gauge transformations of the form (11) is a gauge transformation of the form $(14)[5,9,6]$ :

$$
\left[\delta_{1}, \delta_{2}\right] q^{i} \equiv R_{\gamma}^{i} \epsilon^{\gamma}+E^{i j} L_{j}
$$

where,

$$
\begin{gathered}
\epsilon^{\gamma}=T_{\alpha \beta}^{\gamma}(q, \dot{q}) \epsilon_{1}^{\alpha} \epsilon_{2}^{\beta} \\
T_{\alpha \beta}^{\gamma}=-T_{\beta \alpha}^{\gamma} \\
E^{i j}=E_{\alpha \beta}^{i j}(q, \dot{q}) \epsilon_{1}^{\alpha} \epsilon_{2}^{\beta} \\
E_{\alpha \beta}^{i j}=-E_{\alpha \beta}^{j i}=-E_{\beta \alpha}^{i j}
\end{gathered}
$$

The lagrangian gauge generators $R_{\mu}^{i}$ form an open gauge algebra if $E_{\alpha \beta}^{i j} \neq 0[8,5,9,6,10]$. If $E_{\alpha \beta}^{i j}=0$, the gauge algebra is said to be closed ${ }^{1}$.

The tensors $T_{\alpha \beta}^{\gamma}$ and $E_{\alpha \beta}^{i j}$ are the so-called second-order gauge structure functions of the gauge algebra in the lagrangian formalism. The gauge generators $R_{\mu}^{i}$ are the first-order structure functions of the lagrangian gauge algebra. The zeroth-order structure function is the action $\mathrm{S}$ itself [6].

Since $\epsilon_{1}^{\mu}$ and $\epsilon_{2}^{\nu}$ are arbitrary functions of time, then from (17) we obtain the following identities in the velocity phase space $T Q$ :

$$
\begin{gathered}
\frac{\partial R_{\mu}^{i}}{\partial q^{j}} R_{\nu}^{j}-\frac{\partial R_{\nu}^{i}}{\partial q^{j}} R_{\mu}^{j}+\left(\frac{\partial R_{\mu}^{i}}{\partial \dot{q}^{j}} \frac{\partial R_{\nu}^{j}}{\partial q^{k}}-\frac{\partial R_{\nu}^{i}}{\partial \dot{q}^{j}} \frac{\partial R_{\mu}^{j}}{\partial q^{k}}\right) \dot{q}^{k} \equiv T_{\mu \nu}^{\gamma} R_{\gamma}^{i}-E_{\mu \nu}^{i j} \alpha_{j} \\
\frac{\partial R_{\mu}^{i}}{\partial \dot{q}^{j}} \frac{\partial R_{\nu}^{j}}{\partial \dot{q}^{k}}-\frac{\partial R_{\nu}^{i}}{\partial \dot{q}^{j}} \frac{\partial R_{\mu}^{j}}{\partial \dot{q}^{k}} \equiv E_{\mu \nu}^{i j} W_{j k}
\end{gathered}
$$

\footnotetext{
${ }^{1}$ Notice that by adding trivial gauge transformations to the non-trivial gauge transformations (11) a closed gauge algebra can easily be converted into an "open" gauge algebra. However, in this case the algebra is not really open but "hidden closed". I would like to thank the referee for pointing this out.
} 


$$
\frac{\partial R_{\mu}^{i}}{\partial \dot{q}^{j}} R_{\nu}^{j} \equiv 0
$$

It is not difficult to prove that the identities (22-24) are equivalent to the so-called second-order relations of the lagrangian gauge algebra given in $[6,10]$.

From $(5,6)$ and $(24)$ it follows [11] that there exist some functions $b_{\mu}^{i j}(q, \dot{q})$ such that:

$$
\frac{\partial R_{\mu}^{i}}{\partial \dot{q}^{j}} \equiv b_{\mu}^{i k} W_{k j}
$$

Substituting (25) into (23) we find the following identities:

$$
\left(E_{\mu \nu}^{i j}-b_{\mu}^{i m} W_{m n} b_{\nu}^{n j}+b_{\nu}^{i m} W_{m n} b_{\mu}^{n j}\right) W_{j k} \equiv 0
$$

From (6), (9), (22) and (23) one can see that the structure functions $E_{\mu \nu}^{i j}$ are not uniquely determined. In general, one can write:

$$
E_{\mu \nu}^{i j}=b_{\mu}^{i m} W_{m n} b_{\nu}^{n j}-b_{\nu}^{i m} W_{m n} b_{\mu}^{n j}+e_{\mu \nu}^{\alpha \beta}\left(R_{\alpha}^{i} R_{\beta}^{j}-R_{\beta}^{i} R_{\alpha}^{j}\right)
$$

where $e_{\mu \nu}^{\alpha \beta}(q, \dot{q})$ are arbitrary functions on $T Q$ that are antisymmetric in the lower indexes:

$$
e_{\mu \nu}^{\alpha \beta}=-e_{\nu \mu}^{\alpha \beta}
$$

From (25) and (27) it immediately follows that:

$$
\frac{\partial R_{\alpha}^{i}}{\partial \dot{q}^{k}} E_{\beta \gamma}^{k j}+\frac{\partial R_{\beta}^{i}}{\partial \dot{q}^{k}} E_{\gamma \alpha}^{k j}+\frac{\partial R_{\gamma}^{i}}{\partial \dot{q}^{k}} E_{\alpha \beta}^{k j}+\frac{\partial R_{\alpha}^{j}}{\partial \dot{q}^{k}} E_{\beta \gamma}^{k i}+\frac{\partial R_{\beta}^{j}}{\partial \dot{q}^{k}} E_{\gamma \alpha}^{k i}+\frac{\partial R_{\gamma}^{j}}{\partial \dot{q}^{k}} E_{\alpha \beta}^{k i} \equiv 0
$$

The third-order relations of the lagrangian gauge algebra can be derived from the Jacobi identities $[5,9,6,10]$ :

$$
\left[\delta_{1},\left[\delta_{2}, \delta_{3}\right]\right]+\left[\delta_{2},\left[\delta_{3}, \delta_{1}\right]\right]+\left[\delta_{3},\left[\delta_{1}, \delta_{2}\right]\right] \equiv 0
$$

From (30), using $(11,12,17-24)$ and (29), we obtain the following identities:

$$
\begin{gathered}
R_{\rho}^{i} A_{\alpha \beta \gamma}^{\rho}+B_{\alpha \beta \gamma}^{i j} L_{j} \equiv 0 \\
-R_{\eta}^{i} R_{\alpha}^{k} \frac{\partial T_{\beta \gamma}^{\eta}}{\partial \dot{q}^{k}}-\frac{\partial R_{\eta}^{i}}{\partial \dot{q}^{k}} R_{\alpha}^{k} T_{\beta \gamma}^{\eta}+\left(\frac{\partial R_{\beta}^{i}}{\partial \dot{q}^{k}} E_{\gamma \alpha}^{k j}+\frac{\partial R_{\gamma}^{i}}{\partial \dot{q}^{k}} E_{\alpha \beta}^{k j}+\frac{\partial R_{\alpha}^{j}}{\partial \dot{q}^{k}} E_{\beta \gamma}^{k i}-R_{\alpha}^{k} \frac{\partial E_{\beta \gamma}^{i j}}{\partial \dot{q}^{k}}\right) L_{j} \equiv 0
\end{gathered}
$$

where, 


$$
\begin{aligned}
& A_{\alpha \beta \gamma}^{\rho} \equiv \frac{1}{3}\left[T_{\alpha \eta}^{\rho} T_{\beta \gamma}^{\eta}+T_{\beta \eta}^{\rho} T_{\gamma \alpha}^{\eta}+T_{\gamma \eta}^{\rho} T_{\alpha \beta}^{\eta}\right. \\
& -R_{\alpha}^{j} \frac{\partial T_{\beta \gamma}^{\rho}}{\partial q^{j}}-R_{\beta}^{j} \frac{\partial T_{\gamma \alpha}^{\rho}}{\partial q^{j}}-R_{\gamma}^{j} \frac{\partial T_{\alpha \beta}^{\rho}}{\partial q^{j}} \\
& \left.-\dot{R}_{\alpha}^{j} \frac{\partial T_{\beta \gamma}^{\rho}}{\partial \dot{q}^{j}}-\dot{R}_{\beta}^{j} \frac{\partial T_{\gamma \alpha}^{\rho}}{\partial \dot{q}^{j}}-\dot{R}_{\gamma}^{j} \frac{\partial T_{\alpha \beta}^{\rho}}{\partial \dot{q}^{j}}\right] \\
& B_{\alpha \beta \gamma}^{i j} \equiv \frac{1}{3}\left[E_{\alpha \eta}^{i j} T_{\beta \gamma}^{\eta}+E_{\beta \eta}^{i j} T_{\gamma \alpha}^{\eta}+E_{\gamma \eta}^{i j} T_{\alpha \beta}^{\eta}\right. \\
& -R_{\alpha}^{k} \frac{\partial E_{\beta \gamma}^{i j}}{\partial q^{k}}-R_{\beta}^{k} \frac{\partial E_{\gamma \alpha}^{i j}}{\partial q^{k}}-R_{\gamma}^{k} \frac{\partial E_{\alpha \beta}^{i j}}{\partial q^{k}}-\dot{R}_{\alpha}^{k} \frac{\partial E_{\beta \gamma}^{i j}}{\partial \dot{q}^{k}}-\dot{R}_{\beta}^{k} \frac{\partial E_{\gamma \alpha}^{i j}}{\partial \dot{q}^{k}}-\dot{R}_{\gamma}^{k} \frac{\partial E_{\alpha \beta}^{i j}}{\partial \dot{q}^{k}} \\
& +\frac{\partial R_{\alpha}^{i}}{\partial q^{k}} E_{\beta \gamma}^{k j}+\frac{\partial R_{\beta}^{i}}{\partial q^{k}} E_{\gamma \alpha}^{k j}+\frac{\partial R_{\gamma}^{i}}{\partial q^{k}} E_{\alpha \beta}^{k j}-\frac{\partial R_{\alpha}^{j}}{\partial q^{k}} E_{\beta \gamma}^{k i}-\frac{\partial R_{\beta}^{j}}{\partial q^{k}} E_{\gamma \alpha}^{k i}-\frac{\partial R_{\gamma}^{j}}{\partial q^{k}} E_{\alpha \beta}^{k i} \\
& +\frac{\partial R_{\alpha}^{i}}{\partial \dot{q}^{k}} \dot{E}_{\beta \gamma}^{k j}+\frac{\partial R_{\beta}^{i}}{\partial \dot{q}^{k}} \dot{E}_{\gamma \alpha}^{k j}+\frac{\partial R_{\gamma}^{i}}{\partial \dot{q}^{k}} \dot{E}_{\alpha \beta}^{k j}-\frac{\partial R_{\alpha}^{j}}{\partial \dot{q}^{k}} \dot{E}_{\beta \gamma}^{k i}-\frac{\partial R_{\beta}^{j}}{\partial \dot{q}^{k}} \dot{E}_{\gamma \alpha}^{k i}-\frac{\partial R_{\gamma}^{j}}{\partial \dot{q}^{k}} \dot{E}_{\alpha \beta}^{k i} \\
& \left.+\frac{1}{2} \frac{d}{d t}\left(\frac{\partial R_{\alpha}^{j}}{\partial \dot{q}^{k}} E_{\beta \gamma}^{k i}+\frac{\partial R_{\beta}^{j}}{\partial \dot{q}^{k}} E_{\gamma \alpha}^{k i}+\frac{\partial R_{\gamma}^{j}}{\partial \dot{q}^{k}} E_{\alpha \beta}^{k i}-\frac{\partial R_{\alpha}^{i}}{\partial \dot{q}^{k}} E_{\beta \gamma}^{k j}-\frac{\partial R_{\beta}^{i}}{\partial \dot{q}^{k}} E_{\gamma \alpha}^{k j}-\frac{\partial R_{\gamma}^{i}}{\partial \dot{q}^{k}} E_{\alpha \beta}^{k j}\right)\right]
\end{aligned}
$$

Since, by assumption, the gauge generators $R_{\rho}^{i}$ are irreducible, then from (31) it follows that there must exist some functions $D_{\alpha \beta \gamma}^{i \rho}$ such that $[6,10]$ :

$$
A_{\alpha \beta \gamma}^{\rho} \equiv D_{\alpha \beta \gamma}^{i \rho} L_{i}
$$

The identities (35) are the so-called third-order relations of the lagrangian gauge algebra [6]. $D_{\alpha \beta \gamma}^{i \rho}$ are the third-order gauge structure functions in the lagrangian formalism.

From (35) we see that the third-order structure functions are not uniquely determined. Indeed, if $D_{\alpha \beta \gamma}^{i \rho}(q, \dot{q})$ satisfy (35), then using (33) and (2) we can rewrite (35) as identities in $T Q$ :

$$
\begin{gathered}
\frac{1}{3}\left[T_{\alpha \eta}^{\rho} T_{\beta \gamma}^{\eta}+T_{\beta \eta}^{\rho} T_{\gamma \alpha}^{\eta}+T_{\gamma \eta}^{\rho} T_{\alpha \beta}^{\eta}-R_{\alpha}^{j} \frac{\partial T_{\beta \gamma}^{\rho}}{\partial q^{j}}-R_{\beta}^{j} \frac{\partial T_{\gamma \alpha}^{\rho}}{\partial q^{j}}-R_{\gamma}^{j} \frac{\partial T_{\alpha \beta}^{\rho}}{\partial q^{j}}\right. \\
\left.-\left(\frac{\partial R_{\alpha}^{j}}{\partial q^{l}} \frac{\partial T_{\beta \gamma}^{\rho}}{\partial \dot{q}^{j}}+\frac{\partial R_{\beta}^{j}}{\partial q^{l}} \frac{\partial T_{\gamma \alpha}^{\rho}}{\partial \dot{q}^{j}}+\frac{\partial R_{\gamma}^{j}}{\partial q^{l}} \frac{\partial T_{\alpha \beta}^{\rho}}{\partial \dot{q}^{j}}\right) \dot{q}^{l}\right] \equiv-D_{\alpha \beta \gamma}^{i \rho} \alpha_{i} \\
-\frac{1}{3}\left[\frac{\partial R_{\alpha}^{j}}{\partial \dot{q}^{k}} \frac{\partial T_{\beta \gamma}^{\rho}}{\partial \dot{q}^{j}}+\frac{\partial R_{\beta}^{j}}{\partial \dot{q}^{k}} \frac{\partial T_{\gamma \alpha}^{\rho}}{\partial \dot{q}^{j}}+\frac{\partial R_{\gamma}^{j}}{\partial \dot{q}^{k}} \frac{\partial T_{\alpha \beta}^{\rho}}{\partial \dot{q}^{j}}\right] \equiv D_{\alpha \beta \gamma}^{i \rho} W_{i k}
\end{gathered}
$$


From $(36,37)$ and $(6,9)$ we conclude that any function $\tilde{D}_{\alpha \beta \gamma}^{i \rho}$ :

$$
\tilde{D}_{\alpha \beta \gamma}^{i \rho}=D_{\alpha \beta \gamma}^{i \rho}+d_{\alpha \beta \gamma}^{\rho \delta} R_{\delta}^{i}
$$

must also be a solution of $(36,37)$. The quantities $d_{\alpha \beta \gamma}^{\rho \delta}$ are arbitrary functions.

Finally, from (31) and (35), using the properties of irreducibility (7) and completeness $(13,14)$, one can obtain the fourth-order relations of the lagrangian gauge algebra in the form $[10]:$

$$
R_{\rho}^{i} D_{\alpha \beta \gamma}^{j \rho}-R_{\rho}^{j} D_{\alpha \beta \gamma}^{i \rho}+B_{\alpha \beta \gamma}^{i j} \equiv M_{\alpha \beta \gamma}^{i j k} L_{k}
$$

The tensors $M_{\alpha \beta \gamma}^{i j k}$ are the fourth-order gauge structure functions in the lagrangian formalism.

Higher order gauge structure relations can be obtained by commuting a higher number of infinitesimal gauge transformations. All the lagrangian gauge structure relations are encoded in the so-called classical master equation obeyed by the field-antifield action functional [6, 10]. The lagrangian gauge algebra is characterized by the whole set of gauge structure functions.

The existence of the lagrangian gauge structure functions $D_{\alpha \beta \gamma}^{i \rho}$ and $M_{\alpha \beta \gamma}^{i j k}$ has been proven using an axiomatic approach [6, 10]. In this paper we aim to construct these functions explicitly.

With this purpose in mind, let us develop the hamiltonian formulation for the action functional (1). From (5) it follows that our system has $m$ independent primary constraints $G_{\mu}(\mu=1,2, \ldots, m)$. Following Dirac's method [12], these primary constraints are obtained from the relations that define the canonical momenta:

$$
p_{i}=\frac{\partial L}{\partial \dot{q}^{i}}(q, \dot{q})
$$

From (40) and (5) we see that:

$$
\operatorname{rank}\left\|\frac{\partial G_{\mu}}{\partial p_{i}}\right\|=m
$$

The set of hamiltonian constraints:

$$
G_{\mu}(q, p)=0
$$

defines a submanifold of the momentum phase space $T^{*} Q$ which is denoted by $\mathcal{M}$.

The canonical Hamiltonian $H_{c}(q, p)$ is any function in the momentum phase space $T^{*} Q$ satisfying the following relation:

$$
\mathrm{FL}^{*} H_{c} \equiv H_{c}\left(q, \frac{\partial L}{\partial \dot{q}}(q, \dot{q})\right)=\dot{q}^{i} \frac{\partial L}{\partial \dot{q}^{i}}-L
$$

Following [13] FL is the application fiber derivative of the Lagrangian of the tangent bundle $T Q$ on the cotangent bundle $T^{*} Q$, 


\section{$\mathrm{FL}: T Q \longrightarrow T^{*} Q$}

given by $\mathrm{FL}(q, \dot{q})=(q, p)$ as defined by $(40)$. $\mathrm{FL}^{*}$ is the pullback application.

For the primary hamiltonian constraints $G_{\mu}$ we have:

$$
\mathrm{FL}^{*} G_{\mu}=G_{\mu}\left(q, \frac{\partial L}{\partial \dot{q}}(q, \dot{q})\right) \equiv 0
$$

Differentiating (44) with respect to $\dot{q}^{j}$ we obtain:

$$
\mathrm{FL}^{*} \frac{\partial G_{\mu}}{\partial p_{i}} W_{i j} \equiv 0
$$

Equations (45), (41), (6) and (7) allow us to identify $\mathrm{FL}^{*} \frac{\partial G_{\mu}}{\partial p_{i}}$ with the irreducible gauge generators $R_{\mu}^{i}$ :

$$
R_{\mu}^{i}(q, \dot{q})=\mathrm{FL}^{*} \frac{\partial G_{\mu}}{\partial p_{i}}
$$

In other words, one can always choose the constraints $G_{\mu}$ in such a way that the relations (46) are true.

From (46) and (44) it also follows that:

$$
-R_{\mu}^{j} \frac{\partial^{2} L}{\partial q^{i} \partial \dot{q}^{j}}=\mathrm{FL}^{*} \frac{\partial G_{\mu}}{\partial q^{i}}
$$

From the definition of the canonical Hamiltonian (43) we can also derive the following relations [11]:

$$
\begin{gathered}
\mathrm{FL}^{*} \frac{\partial H_{c}}{\partial p_{i}}=\dot{q}^{i}-\lambda_{\mu}(q, \dot{q}) R_{\mu}^{i}(q, \dot{q}) \\
\mathrm{FL}^{*} \frac{\partial H_{c}}{\partial q^{i}}=-\frac{\partial L}{\partial q^{i}}+\lambda_{\mu}(q, \dot{q}) R_{\mu}^{j}(q, \dot{q}) \frac{\partial^{2} L}{\partial \dot{q}^{j} \partial q^{i}}
\end{gathered}
$$

Let us consider the Poisson brackets among the primary hamiltonian constraints. From (46) and (47) we obtain:

$$
\mathrm{FL}^{*}\left\{G_{\mu}, G_{\nu}\right\} \equiv-R_{\mu}^{i} B_{i j} R_{\nu}^{j}
$$

where,

$$
B_{i j}=\frac{\partial^{2} L}{\partial \dot{q}^{i} \partial q^{j}}-\frac{\partial^{2} L}{\partial \dot{q}^{j} \partial q^{i}}
$$

For the Poisson brackets between the constraints and the canonical Hamiltonian we have:

$$
\mathrm{FL}^{*}\left\{H_{c}, G_{\mu}\right\} \equiv-R_{\mu}^{i} \alpha_{i}-R_{\mu}^{i} B_{i j} R_{\nu}^{j} \lambda_{\nu}
$$


Differentiating (9) with respect to $\dot{q}^{j}$ and using the identities (6) we obtain the following identities in $T Q$ :

$$
\frac{\partial R_{\alpha}^{i}}{\partial \dot{q}^{j}} \alpha_{i}-R_{\alpha}^{i} B_{i j}+\dot{q}^{l} \frac{\partial R_{\alpha}^{i}}{\partial q^{l}} W_{i j} \equiv 0
$$

Multiplying (53) by $R_{\beta}^{j}$ and using (24) and (6) we obtain the identities:

$$
R_{\alpha}^{i} B_{i j} R_{\beta}^{j} \equiv 0
$$

Therefore, from (50), (52), (9) and (54) we obtain the following identities in the velocity phase space $T Q$ :

$$
\begin{aligned}
& \mathrm{FL}^{*}\left\{G_{\mu}, G_{\nu}\right\} \equiv 0 \\
& \mathrm{FL}^{*}\left\{H_{c}, G_{\mu}\right\} \equiv 0
\end{aligned}
$$

or equivalently, in the cotangent manifold $T^{*} Q$ :

$$
\begin{array}{r}
\left\{G_{\mu}, G_{\nu}\right\} \equiv C_{\mu \nu}^{\alpha} G_{\alpha} \\
\left\{H_{c}, G_{\mu}\right\} \equiv V_{\mu}^{\alpha} G_{\alpha}
\end{array}
$$

where $C_{\mu \nu}^{\alpha}(q, p)$ and $V_{\mu}^{\alpha}(q, p)$ are funtions in $T^{*} Q$.

From the identities (57) we conclude that all the hamiltonian constraints $G_{\mu}(\mu=$ $1,2, \ldots, m)$ are first-class constraints [12]. From (58) we conclude that our system has no secondary constraints $[12]$.

The identities (57) are the first-order relations of the gauge algebra in the hamiltonian formalism $[2,14]$. The constraints $G_{\mu}$ are also called the zeroth-order hamiltonian structure functions $[2,14]$. The first-order hamiltonian structure functions are the functions $C_{\mu \nu}^{\alpha}$ in (57) $[2,14]$.

The second-order relations of the hamiltonian gauge algebra follow from the Jacobi identities:

$$
\left\{\left\{G_{\alpha}, G_{\beta}\right\}, G_{\gamma}\right\}+\left\{\left\{G_{\beta}, G_{\gamma}\right\}, G_{\alpha}\right\}+\left\{\left\{G_{\gamma}, G_{\alpha}\right\}, G_{\beta}\right\} \equiv 0
$$

Indeed, from (59) and (57) it follows that:

$$
\left(\left\{C_{\alpha \beta}^{\eta}, G_{\gamma}\right\}+\left\{C_{\beta \gamma}^{\eta}, G_{\alpha}\right\}+\left\{C_{\gamma \alpha}^{\eta}, G_{\beta}\right\}-C_{\alpha \beta}^{\delta} C_{\gamma \delta}^{\eta}-C_{\beta \gamma}^{\delta} C_{\alpha \delta}^{\eta}-C_{\gamma \alpha}^{\delta} C_{\beta \delta}^{\eta}\right) G_{\eta} \equiv 0
$$

From the irreducibility of the constraints (41) it follows [2] that there must exist some functions $J_{\alpha \beta \gamma}^{\eta \sigma}$ in the momentum phase space $T^{*} Q$ such that:

$$
\left\{C_{\alpha \beta}^{\eta}, G_{\gamma}\right\}+\left\{C_{\beta \gamma}^{\eta}, G_{\alpha}\right\}+\left\{C_{\gamma \alpha}^{\eta}, G_{\beta}\right\}-C_{\alpha \beta}^{\delta} C_{\gamma \delta}^{\eta}-C_{\beta \gamma}^{\delta} C_{\alpha \delta}^{\eta}-C_{\gamma \alpha}^{\delta} C_{\beta \delta}^{\eta} \equiv J_{\alpha \beta \gamma}^{\eta \sigma} G_{\sigma}
$$


or equivalently:

$$
\mathrm{FL}^{*}\left(\left\{C_{\alpha \beta}^{\eta}, G_{\gamma}\right\}+\left\{C_{\beta \gamma}^{\eta}, G_{\alpha}\right\}+\left\{C_{\gamma \alpha}^{\eta}, G_{\beta}\right\}-C_{\alpha \beta}^{\delta} C_{\gamma \delta}^{\eta}-C_{\beta \gamma}^{\delta} C_{\alpha \delta}^{\eta}-C_{\gamma \alpha}^{\delta} C_{\beta \delta}^{\eta}\right) \equiv 0
$$

The identities (61) are the so-called second-order relations of the gauge algebra in the hamiltonian formalism $[2,14]$. The functions $J_{\alpha \beta \gamma}^{\eta \sigma}$ are the second-order hamiltonian structure functions.

Our purpose in this paper is to write the lagrangian gauge structure functions $\left(R_{\mu}^{i}, T_{\alpha \beta}^{\gamma}\right.$, $E_{\alpha \beta}^{i j}, D_{\alpha \beta \gamma}^{i \rho}$ and $\left.M_{\alpha \beta \gamma}^{i j k}\right)$ explicitly in terms of the hamiltonian structure functions $G_{\mu}, C_{\alpha \beta}^{\gamma}$ and their derivatives. As we shall see, knowledge of the functional dependence of $J_{\alpha \beta \gamma}^{\eta \sigma}$ or other higher order hamiltonian structure functions is not required for this.

Equations (46) give us the lagrangian gauge generators $R_{\mu}^{i}$.

From (46), (40) and (3) it immediately follows that:

$$
\begin{gathered}
\frac{\partial R_{\mu}^{i}}{\partial \dot{q}^{k}} \equiv W_{k l} \mathrm{FL}^{*} \frac{\partial^{2} G_{\mu}}{\partial p_{l} \partial p_{i}} \\
\frac{\partial R_{\mu}^{i}}{\partial q^{k}} \equiv \mathrm{FL}^{*} \frac{\partial^{2} G_{\mu}}{\partial q^{k} \partial p_{i}}+\frac{\partial^{2} L}{\partial q^{k} \partial \dot{q}^{l}} \mathrm{FL}^{*} \frac{\partial^{2} G_{\mu}}{\partial p_{l} \partial p_{i}}
\end{gathered}
$$

Substituting $(63,64)$ into $(22-24)$ we find:

$$
\begin{gathered}
\mathrm{FL}^{*}\left(\frac{\partial^{2} G_{\mu}}{\partial p_{i} \partial q^{j}} \frac{\partial G_{\nu}}{\partial p_{j}}-\frac{\partial^{2} G_{\nu}}{\partial p_{i} \partial q^{j}} \frac{\partial G_{\mu}}{\partial p_{j}}\right)+\mathrm{FL}^{*}\left(\frac{\partial^{2} G_{\mu}}{\partial p_{i} \partial p_{l}} \frac{\partial G_{\nu}}{\partial p_{j}}-\frac{\partial^{2} G_{\nu}}{\partial p_{i} \partial p_{l}} \frac{\partial G_{\mu}}{\partial p_{j}}\right) \frac{\partial^{2} L}{\partial \dot{q}^{l} \partial q^{j}}+ \\
+\mathrm{FL}^{*}\left(\frac{\partial^{2} G_{\mu}}{\partial p_{i} \partial p_{l}} \frac{\partial^{2} G_{\nu}}{\partial p_{m} \partial p_{j}}-\frac{\partial^{2} G_{\nu}}{\partial p_{i} \partial p_{l}} \frac{\partial^{2} G_{\mu}}{\partial p_{m} \partial p_{j}}\right) W_{l m} \frac{\partial^{2} L}{\partial \dot{q}^{j} \partial q^{k}} \dot{q}^{k}+ \\
+\mathrm{FL}^{*}\left(\frac{\partial^{2} G_{\mu}}{\partial p_{i} \partial p_{l}} \frac{\partial^{2} G_{\nu}}{\partial p_{m} \partial q^{k}}-\frac{\partial^{2} G_{\nu}}{\partial p_{i} \partial p_{l}} \frac{\partial^{2} G_{\mu}}{\partial p_{m} \partial q^{k}}\right) \dot{q}^{k} W_{l m} \equiv T_{\mu \nu}^{\gamma} R_{\gamma}^{i}-E_{\mu \nu}^{i j} \alpha_{j} \\
\left(\mathrm{FL}^{*} \frac{\partial^{2} G_{\mu}}{\partial p_{i} \partial p_{l}} W_{l m} \mathrm{FL}^{*} \frac{\partial^{2} G_{\nu}}{\partial p_{m} \partial p_{j}}-\mathrm{FL}^{*} \frac{\partial^{2} G_{\nu}}{\partial p_{i} \partial p_{l}} W_{l m} \mathrm{FL}^{*} \frac{\partial^{2} G_{\mu}}{\partial p_{m} \partial p_{j}}\right) W_{j k} \equiv E_{\mu \nu}^{i j} W_{j k} \\
\mathrm{FL}^{*} \frac{\partial^{2} G_{\mu}}{\partial p_{i} \partial p_{k}} W_{k j} R_{\nu}^{j} \equiv 0
\end{gathered}
$$

Substituting (51) and $(46,47)$ into (65) and using (4) we see that the identities (65) can be rewritten as:

$$
\begin{array}{r}
\mathrm{FL}^{*} \frac{\partial}{\partial p_{i}}\left\{G_{\mu}, G_{\nu}\right\}-\left(\mathrm{FL}^{*} \frac{\partial^{2} G_{\mu}}{\partial p_{i} \partial p_{l}} W_{l m} \mathrm{FL}^{*} \frac{\partial^{2} G_{\nu}}{\partial p_{m} \partial p_{j}}-\mathrm{FL}^{*} \frac{\partial^{2} G_{\nu}}{\partial p_{i} \partial p_{l}} W_{l m} \mathrm{FL}^{*} \frac{\partial^{2} G_{\mu}}{\partial p_{m} \partial p_{j}}\right) \alpha_{j} \\
+\mathrm{FL}^{*} \frac{\partial^{2} G_{\mu}}{\partial p_{i} \partial_{l}}\left(B_{l j} \mathrm{FL}^{*} \frac{\partial G_{\nu}}{\partial p_{j}}+W_{l m} \mathrm{FL}^{*} \frac{\partial^{2} G_{\nu}}{\partial p_{m} \partial p_{j}} \frac{\partial L}{\partial q^{j}}+W_{l m} \mathrm{FL}^{*} \frac{\partial^{2} G_{\nu}}{\partial p_{m} \partial q^{k}} \dot{q}^{k}\right)
\end{array}
$$




$$
\begin{array}{r}
-\mathrm{FL}^{*} \frac{\partial^{2} G_{\nu}}{\partial p_{i} \partial_{l}}\left(B_{l j} \mathrm{FL}^{*} \frac{\partial G_{\mu}}{\partial p_{j}}+W_{l m} \mathrm{FL}^{*} \frac{\partial^{2} G_{\mu}}{\partial p_{m} \partial p_{j}} \frac{\partial L}{\partial q^{j}}+W_{l m} \mathrm{FL}^{*} \frac{\partial^{2} G_{\mu}}{\partial p_{m} \partial q^{k}} \dot{q}^{k}\right) \\
\equiv T_{\mu \nu}^{\gamma} R_{\gamma}^{i}-E_{\mu \nu}^{i j} \alpha_{j}
\end{array}
$$

Notice that:

$$
\mathrm{FL}^{*} \frac{\partial}{\partial p_{i}}\left\{G_{\mu}, G_{\nu}\right\} \equiv \mathrm{FL}^{*}\left(\frac{\partial C_{\mu \nu}^{\gamma}}{\partial p_{i}} G_{\gamma}+C_{\mu \nu}^{\gamma} \frac{\partial G_{\gamma}}{\partial p_{i}}\right) \equiv \mathrm{FL}^{*} C_{\mu \nu}^{\gamma} \mathrm{FL}^{*} \frac{\partial G_{\gamma}}{\partial p_{i}}
$$

On the other hand, from (53), (63) and (64) it follows that:

$$
B_{l j} \mathrm{FL}^{*} \frac{\partial G_{\alpha}}{\partial p_{j}}+W_{l m} \mathrm{FL}^{*} \frac{\partial^{2} G_{\alpha}}{\partial p_{m} \partial p_{j}} \frac{\partial L}{\partial q^{j}}+W_{l m} \mathrm{FL}^{*} \frac{\partial^{2} G_{\alpha}}{\partial p_{m} \partial q^{k}} \dot{q}^{k} \equiv 0
$$

Finally, substituting (69) and (70) into (68) we obtain the identities:

$$
\begin{aligned}
\mathrm{FL}^{*} C_{\mu \nu}^{\gamma} R_{\gamma}^{i}-\left(\mathrm{FL}^{*} \frac{\partial^{2} G_{\mu}}{\partial p_{i} \partial p_{l}} W_{l m} \mathrm{FL}^{*} \frac{\partial^{2} G_{\nu}}{\partial p_{m} \partial p_{j}}-\mathrm{FL}^{*} \frac{\partial^{2} G_{\nu}}{\partial p_{i} \partial p_{l}} W_{l m} \mathrm{FL}^{*} \frac{\partial^{2} G_{\mu}}{\partial p_{m} \partial p_{j}}\right) \alpha_{j} \\
\equiv T_{\mu \nu}^{\gamma} R_{\gamma}^{i}-E_{\mu \nu}^{i j} \alpha_{j}
\end{aligned}
$$

From the identities (71) and (66) it follows that we can make the following identification:

$$
\begin{gathered}
T_{\mu \nu}^{\gamma}=\mathrm{FL}^{*} C_{\mu \nu}^{\gamma} \\
E_{\mu \nu}^{i j}=\mathrm{FL}^{*} \frac{\partial^{2} G_{\mu}}{\partial p_{i} \partial p_{l}} W_{l m} \mathrm{FL}^{*} \frac{\partial^{2} G_{\nu}}{\partial p_{m} \partial p_{j}}-\mathrm{FL}^{*} \frac{\partial^{2} G_{\nu}}{\partial p_{i} \partial p_{l}} W_{l m} \mathrm{FL}^{*} \frac{\partial^{2} G_{\mu}}{\partial p_{m} \partial p_{j}}
\end{gathered}
$$

Equations (72) and (73) give us the lagrangian second-order gauge structure functions in terms of the hamiltonian first-order structure functions and the second derivatives of the hamiltonian constraints with respect to the canonical momenta.

Our task now is to find an expression for the third-order lagrangian gauge structure functions $D_{\alpha \beta \gamma}^{i \rho}$. From (72) it follows that:

$$
\begin{gathered}
\frac{\partial T_{\alpha \beta}^{\rho}}{\partial q^{j}}=\mathrm{FL}^{*} \frac{\partial C_{\alpha \beta}^{\rho}}{\partial q^{j}}+\frac{\partial^{2} L}{\partial q^{j} \partial \dot{q}^{k}} \mathrm{FL}^{*} \frac{\partial C_{\alpha \beta}^{\rho}}{\partial p_{k}} \\
\frac{\partial T_{\alpha \beta}^{\rho}}{\partial \dot{q}^{j}}=W_{j k} \mathrm{FL}^{*} \frac{\partial C_{\alpha \beta}^{\rho}}{\partial p_{k}}
\end{gathered}
$$

Notice also that using $(63,64)$ and $(2),(4)$ we can write:

$$
\dot{R}_{\alpha}^{j}=\mathrm{FL}^{*} \frac{\partial^{2} G_{\alpha}}{\partial p_{j} \partial q^{l}} \dot{q}^{l}+\mathrm{FL}^{*} \frac{\partial^{2} G_{\alpha}}{\partial p_{j} \partial p_{l}} \frac{\partial L}{\partial q^{l}}+\mathrm{FL}^{*} \frac{\partial^{2} G_{\alpha}}{\partial p_{j} \partial p_{k}} L_{k}
$$

Substituting (72), (74), (75) and (76) into (33) we obtain: 


$$
\begin{aligned}
A_{\alpha \beta \gamma}^{\delta}= & \frac{1}{3}\left[\mathrm{FL}^{*}\left(C_{\alpha \eta}^{\rho} C_{\beta \gamma}^{\eta}+C_{\beta \eta}^{\rho} C_{\gamma \alpha}^{\eta}+C_{\gamma \eta}^{\rho} C_{\alpha \beta}^{\eta}\right)-\mathrm{FL}^{*}\left(\frac{\partial G_{\alpha}}{\partial p_{j}} \frac{\partial C_{\beta \gamma}^{\rho}}{\partial q^{j}}+\frac{\partial G_{\beta}}{\partial p_{j}} \frac{\partial C_{\gamma \alpha}^{\rho}}{\partial q^{j}}+\frac{\partial G_{\gamma}}{\partial p_{j}} \frac{\partial C_{\alpha \beta}^{\rho}}{\partial q^{j}}\right)\right. \\
& -\frac{\partial^{2} L}{\partial q^{j} \partial \dot{q}^{k}} \mathrm{FL}^{*}\left(\frac{\partial G_{\alpha}}{\partial p_{j}} \frac{\partial C_{\beta \gamma}^{\rho}}{\partial p_{k}}+\frac{\partial G_{\beta}}{\partial p_{j}} \frac{\partial C_{\gamma \alpha}^{\rho}}{\partial p_{k}}+\frac{\partial G_{\gamma}}{\partial p_{j}} \frac{\partial C_{\alpha \beta}^{\rho}}{\partial p_{k}}\right) \\
& -\dot{q}^{l}\left(\mathrm{FL}^{*} \frac{\partial^{2} G_{\alpha}}{\partial q^{l} \partial p_{j}} W_{j k} \mathrm{FL}^{*} \frac{\partial C_{\beta \gamma}^{\rho}}{\partial p_{k}}+\mathrm{FL}^{*} \frac{\partial^{2} G_{\beta}}{\partial q^{l} \partial p_{j}} W_{j k} \mathrm{FL}^{*} \frac{\partial C_{\gamma \alpha}^{\rho}}{\partial p_{k}}+\mathrm{FL}^{*} \frac{\partial^{2} G_{\gamma}}{\partial q^{l} \partial p_{j}} W_{j k} \mathrm{FL}^{*} \frac{\partial C_{\alpha \beta}^{\rho}}{\partial p_{k}}\right) \\
- & \frac{\partial L}{\partial q^{l}}\left(\mathrm{FL}^{*} \frac{\partial^{2} G_{\alpha}}{\partial p_{l} \partial p_{j}} W_{j k} \mathrm{FL}^{*} \frac{\partial C_{\beta \gamma}^{\rho}}{\partial p_{k}}+\mathrm{FL}^{*} \frac{\partial^{2} G_{\beta}}{\partial p_{l} \partial p_{j}} W_{j k} \mathrm{FL}^{*} \frac{\partial C_{\gamma \alpha}^{\rho}}{\partial p_{k}}+\mathrm{FL}^{*} \frac{\partial^{2} G_{\gamma}}{\partial p_{l} \partial p_{j}} W_{j k} \mathrm{FL}^{*} \frac{\partial C_{\alpha \beta}^{\rho}}{\partial p_{k}}\right) \\
- & \left.L_{j}\left(\mathrm{FL}^{*} \frac{\partial^{2} G_{\alpha}}{\partial p_{j} \partial p_{l}} W_{l k} \mathrm{FL}^{*} \frac{\partial C_{\beta \gamma}^{\rho}}{\partial p_{k}}+\mathrm{FL}^{*} \frac{\partial^{2} G_{\beta}}{\partial p_{j} \partial p_{l}} W_{l k} \mathrm{FL}^{*} \frac{\partial C_{\gamma \alpha}^{\rho}}{\partial p_{k}}+\mathrm{FL}^{*} \frac{\partial^{2} G_{\gamma}}{\partial p_{j} \partial p_{l}} W_{l k} \mathrm{FL}^{*} \frac{\partial C_{\alpha \beta}^{\rho}}{\partial p_{k}}\right)\right]
\end{aligned}
$$

Using (51) and $(46,47)$ we can rewrite $(77)$ as follows:

$$
\begin{array}{r}
A_{\alpha \beta \gamma}^{\delta}=\frac{1}{3}\left[\mathrm{FL}^{*}\left(-\left\{C_{\alpha \beta}^{\rho}, G_{\gamma}\right\}-\left\{C_{\gamma \alpha}^{\rho}, G_{\beta}\right\}-\left\{C_{\beta \gamma}^{\rho}, G_{\alpha}\right\}+C_{\alpha \eta}^{\rho} C_{\beta \gamma}^{\eta}+C_{\beta \eta}^{\rho} C_{\gamma \alpha}^{\eta}+C_{\gamma \eta}^{\rho} C_{\alpha \beta}^{\eta}\right)\right. \\
-\mathrm{FL}^{*} \frac{\partial C_{\alpha \beta}^{\rho}}{\partial p_{k}}\left(B_{k j} \mathrm{FL}^{*} \frac{\partial G_{\gamma}}{\partial p_{j}}+W_{j k} \mathrm{FL}^{*} \frac{\partial^{2} G_{\gamma}}{\partial p_{l} \partial p_{j}} \frac{\partial L}{\partial q^{l}}+W_{j k} \mathrm{FL}^{*} \frac{\partial^{2} G_{\gamma}}{\partial p_{j} \partial q^{l}} \dot{q}^{l}\right) \\
-\mathrm{FL}^{*} \frac{\partial C_{\gamma \alpha}^{\rho}}{\partial p_{k}}\left(B_{k j} \mathrm{FL}^{*} \frac{\partial G_{\beta}}{\partial p_{j}}+W_{j k} \mathrm{FL}^{*} \frac{\partial^{2} G_{\beta}}{\partial p_{l} \partial p_{j}} \frac{\partial L}{\partial q^{l}}+W_{j k} \mathrm{FL}^{*} \frac{\partial^{2} G_{\beta}}{\partial p_{j} \partial q^{l}} \dot{q}^{l}\right) \\
-\mathrm{FL}^{*} \frac{\partial C_{\beta \gamma}^{\rho}}{\partial p_{k}}\left(B_{k j} \mathrm{FL}^{*} \frac{\partial G_{\alpha}}{\partial p_{j}}+W_{j k} \mathrm{FL}^{*} \frac{\partial^{2} G_{\alpha}}{\partial p_{l} \partial p_{j}} \frac{\partial L}{\partial q^{l}}+W_{j k} \mathrm{FL}^{*} \frac{\partial^{2} G_{\alpha}}{\partial p_{j} \partial q^{l}} \dot{l}^{l}\right) \\
\left.-L_{j}\left(\mathrm{FL}^{*} \frac{\partial^{2} G_{\alpha}}{\partial p_{j} \partial p_{l}} W_{l k} \mathrm{FL}^{*} \frac{\partial C_{\beta \gamma}^{\rho}}{\partial p_{k}}+\mathrm{FL}^{*} \frac{\partial^{2} G_{\beta}}{\partial p_{j} \partial p_{l}} W_{l k} \mathrm{FL}^{*} \frac{\partial C_{\gamma \alpha}^{\rho}}{\partial p_{k}}+\mathrm{FL}^{*} \frac{\partial^{2} G_{\gamma}}{\partial p_{j} \partial p_{l}} W_{l k} \mathrm{FL}^{*} \frac{\partial C_{\alpha \beta}^{\rho}}{\partial p_{k}}\right)\right]
\end{array}
$$

Finally, using (62) and (70) we obtain:

$A_{\alpha \beta \gamma}^{\delta}=-\frac{1}{3} L_{j}\left(\mathrm{FL}^{*} \frac{\partial^{2} G_{\alpha}}{\partial p_{j} \partial p_{l}} W_{l k} \mathrm{FL}^{*} \frac{\partial C_{\beta \gamma}^{\rho}}{\partial p_{k}}+\mathrm{FL}^{*} \frac{\partial^{2} G_{\beta}}{\partial p_{j} \partial p_{l}} W_{l k} \mathrm{FL}^{*} \frac{\partial C_{\gamma \alpha}^{\rho}}{\partial p_{k}}+\mathrm{FL}^{*} \frac{\partial^{2} G_{\gamma}}{\partial p_{j} \partial p_{l}} W_{l k} \mathrm{FL}^{*} \frac{\partial C_{\alpha \beta}^{\rho}}{\partial p_{k}}\right)$

From (35) and (79) we see that the third-order lagrangian gauge structure functions $D_{\alpha \beta \gamma}^{i \rho}$ can be written as:

$$
D_{\alpha \beta \gamma}^{i \rho}=-\frac{1}{3}\left(\mathrm{FL}^{*} \frac{\partial^{2} G_{\alpha}}{\partial p_{i} \partial p_{j}} W_{j k} \mathrm{FL}^{*} \frac{\partial C_{\beta \gamma}^{\rho}}{\partial p_{k}}+\mathrm{FL}^{*} \frac{\partial^{2} G_{\beta}}{\partial p_{i} \partial p_{j}} W_{j k} \mathrm{FL}^{*} \frac{\partial C_{\gamma \alpha}^{\rho}}{\partial p_{k}}+\mathrm{FL}^{*} \frac{\partial^{2} G_{\gamma}}{\partial p_{i} \partial p_{j}} W_{j k} \mathrm{FL}^{*} \frac{\partial C_{\alpha \beta}^{\rho}}{\partial p_{k}}\right)
$$


The derivation presented above can also be viewed as a proof by construction of the existence of the gauge structure functions $D_{\alpha \beta \gamma}^{i \rho}$.

Using (6), (63), (73) and (75) one can easily prove the identities (32).

Let us now find the fourth-order lagrangian gauge structure functions $M_{\alpha \beta \gamma}^{i j k}$. For that, we need to write the left-hand-side of (39) in terms of hamiltonian quantities. After some lengthy calculations we find:

$$
\begin{aligned}
& R_{\rho}^{i} D_{\alpha \beta \gamma}^{j \rho}-R_{\rho}^{j} D_{\alpha \beta \gamma}^{i \rho} \equiv \\
& -\frac{1}{3}\left[E_{\alpha \eta}^{i j} T_{\beta \gamma}^{\eta}+E_{\beta \eta}^{i j} T_{\gamma \alpha}^{\eta}+E_{\gamma \eta}^{i j} T_{\alpha \beta}^{\eta}\right. \\
& +\mathrm{FL}^{*}\left(\frac{\partial^{2}}{\partial p_{i} \partial p_{k}}\left\{G_{\alpha}, G_{\beta}\right\} \frac{\partial^{2} G_{\gamma}}{\partial p_{l} \partial p_{j}}-\frac{\partial^{2}}{\partial p_{j} \partial p_{k}}\left\{G_{\alpha}, G_{\beta}\right\} \frac{\partial^{2} G_{\gamma}}{\partial p_{l} \partial p_{i}}\right) W_{k l} \\
& +\mathrm{FL}^{*}\left(\frac{\partial^{2}}{\partial p_{i} \partial p_{k}}\left\{G_{\beta}, G_{\gamma}\right\} \frac{\partial^{2} G_{\alpha}}{\partial p_{l} \partial p_{j}}-\frac{\partial^{2}}{\partial p_{j} \partial p_{k}}\left\{G_{\beta}, G_{\gamma}\right\} \frac{\partial^{2} G_{\alpha}}{\partial p_{l} \partial p_{i}}\right) W_{k l} \\
& \left.+\mathrm{FL}^{*}\left(\frac{\partial^{2}}{\partial p_{i} \partial p_{k}}\left\{G_{\gamma}, G_{\alpha}\right\} \frac{\partial^{2} G_{\beta}}{\partial p_{l} \partial p_{j}}-\frac{\partial^{2}}{\partial p_{j} \partial p_{k}}\left\{G_{\gamma}, G_{\alpha}\right\} \frac{\partial^{2} G_{\beta}}{\partial p_{l} \partial p_{j}}\right) W_{k l}\right] \\
& B_{\alpha \beta \gamma}^{i j} \equiv \frac{1}{3}\left[E_{\alpha \eta}^{i j} T_{\beta \gamma}^{\eta}+E_{\beta \eta}^{i j} T_{\gamma \alpha}^{\eta}+E_{\gamma \eta}^{i j} T_{\alpha \beta}^{\eta}\right. \\
& +\mathrm{FL}^{*}\left(\frac{\partial^{2}}{\partial p_{i} \partial p_{k}}\left\{G_{\alpha}, G_{\beta}\right\} \frac{\partial^{2} G_{\gamma}}{\partial p_{l} \partial p_{j}}-\frac{\partial^{2}}{\partial p_{j} \partial p_{k}}\left\{G_{\alpha}, G_{\beta}\right\} \frac{\partial^{2} G_{\gamma}}{\partial p_{l} \partial p_{i}}\right) W_{k l} \\
& +\mathrm{FL}^{*}\left(\frac{\partial^{2}}{\partial p_{i} \partial p_{k}}\left\{G_{\beta}, G_{\gamma}\right\} \frac{\partial^{2} G_{\alpha}}{\partial p_{l} \partial p_{j}}-\frac{\partial^{2}}{\partial p_{j} \partial p_{k}}\left\{G_{\beta}, G_{\gamma}\right\} \frac{\partial^{2} G_{\alpha}}{\partial p_{l} \partial p_{i}}\right) W_{k l} \\
& \left.+\mathrm{FL}^{*}\left(\frac{\partial^{2}}{\partial p_{i} \partial p_{k}}\left\{G_{\gamma}, G_{\alpha}\right\} \frac{\partial^{2} G_{\beta}}{\partial p_{l} \partial p_{j}}-\frac{\partial^{2}}{\partial p_{j} \partial p_{k}}\left\{G_{\gamma}, G_{\alpha}\right\} \frac{\partial^{2} G_{\beta}}{\partial p_{l} \partial p_{j}}\right) W_{k l}\right] \\
& -\frac{1}{3} L_{k}\left[\mathrm{FL}^{*} \frac{\partial^{2} G_{\alpha}}{\partial p_{k} \partial p_{l}} \frac{\partial E_{\beta \gamma}^{i j}}{\partial \dot{q}^{l}}+\mathrm{FL}^{*} \frac{\partial^{2} G_{\beta}}{\partial p_{k} \partial p_{l}} \frac{\partial E_{\gamma \alpha}^{i j}}{\partial \dot{q}^{l}}+\mathrm{FL}^{*} \frac{\partial^{2} G_{\gamma}}{\partial p_{k} \partial p_{l}} \frac{\partial E_{\alpha \beta}^{i j}}{\partial \dot{q}^{l}}\right. \\
& +\mathrm{FL}^{*} \frac{\partial^{3} G_{\alpha}}{\partial p_{k} \partial p_{m} \partial p_{n}}\left(\frac{\partial R_{\beta}^{i}}{\partial \dot{q}^{m}} \frac{\partial R_{\gamma}^{j}}{\partial \dot{q}^{n}}-\frac{\partial R_{\gamma}^{i}}{\partial \dot{q}^{m}} \frac{\partial R_{\beta}^{j}}{\partial \dot{q}^{n}}\right) \\
& +\mathrm{FL}^{*} \frac{\partial^{3} G_{\beta}}{\partial p_{k} \partial p_{m} \partial p_{n}}\left(\frac{\partial R_{\gamma}^{i}}{\partial \dot{q}^{m}} \frac{\partial R_{\alpha}^{j}}{\partial \dot{q}^{n}}-\frac{\partial R_{\alpha}^{i}}{\partial \dot{q}^{m}} \frac{\partial R_{\gamma}^{j}}{\partial \dot{q}^{n}}\right) \\
& \left.+\mathrm{FL}^{*} \frac{\partial^{3} G_{\gamma}}{\partial p_{k} \partial p_{m} \partial p_{n}}\left(\frac{\partial R_{\alpha}^{i}}{\partial \dot{q}^{m}} \frac{\partial R_{\beta}^{j}}{\partial \dot{q}^{n}}-\frac{\partial R_{\beta}^{i}}{\partial \dot{q}^{m}} \frac{\partial R_{\alpha}^{j}}{\partial \dot{q}^{n}}\right)\right]
\end{aligned}
$$

From $(39)$ and $(81,82)$ we see that it is possible to make the following identification: 


$$
\begin{aligned}
M_{\alpha \beta \gamma}^{i j k} \equiv & -\frac{1}{3}\left[\mathrm{FL}^{*} \frac{\partial^{2} G_{\alpha}}{\partial p_{k} \partial p_{l}} \frac{\partial E_{\beta \gamma}^{i j}}{\partial \dot{q}^{l}}+\mathrm{FL}^{*} \frac{\partial^{2} G_{\beta}}{\partial p_{k} \partial p_{l}} \frac{\partial E_{\gamma \alpha}^{i j}}{\partial \dot{q}^{l}}+\mathrm{FL}^{*} \frac{\partial^{2} G_{\gamma}}{\partial p_{k} \partial p_{l}} \frac{\partial E_{\alpha \beta}^{i j}}{\partial \dot{q}^{l}}\right. \\
& +\mathrm{FL}^{*} \frac{\partial^{3} G_{\alpha}}{\partial p_{k} \partial p_{m} \partial p_{n}}\left(\frac{\partial R_{\beta}^{i}}{\partial \dot{q}^{m}} \frac{\partial R_{\gamma}^{j}}{\partial \dot{q}^{n}}-\frac{\partial R_{\gamma}^{i}}{\partial \dot{q}^{m}} \frac{\partial R_{\beta}^{j}}{\partial \dot{q}^{n}}\right) \\
& +\mathrm{FL}^{*} \frac{\partial^{3} G_{\beta}}{\partial p_{k} \partial p_{m} \partial p_{n}}\left(\frac{\partial R_{\gamma}^{i}}{\partial \dot{q}^{m}} \frac{\partial R_{\alpha}^{j}}{\partial \dot{q}^{n}}-\frac{\partial R_{\alpha}^{i}}{\partial \dot{q}^{m}} \frac{\partial R_{\gamma}^{j}}{\partial \dot{q}^{n}}\right) \\
& \left.+\mathrm{FL}^{*} \frac{\partial^{3} G_{\gamma}}{\partial p_{k} \partial p_{m} \partial p_{n}}\left(\frac{\partial R_{\alpha}^{i}}{\partial \dot{q}^{m}} \frac{\partial R_{\beta}^{j}}{\partial \dot{q}^{n}}-\frac{\partial R_{\beta}^{i}}{\partial \dot{q}^{m}} \frac{\partial R_{\alpha}^{j}}{\partial \dot{q}^{n}}\right)\right]
\end{aligned}
$$

Using (63) and (73) we can rewrite (83) in terms of the hamiltonian constraints as follows:

$$
\begin{aligned}
M_{\alpha \beta \gamma}^{i j k} \equiv & -\frac{1}{3}\left[\mathrm{FL}^{*} \frac{\partial^{2} G_{\alpha}}{\partial p_{k} \partial p_{l}} P_{l \beta \gamma}^{i j}+\mathrm{FL}^{*} \frac{\partial^{2} G_{\beta}}{\partial p_{k} \partial p_{l}} P_{l \gamma \alpha}^{i j}+\mathrm{FL}^{*} \frac{\partial^{2} G_{\gamma}}{\partial p_{k} \partial p_{l}} P_{l \alpha \beta}^{i j}\right. \\
& +\mathrm{FL}^{*} \frac{\partial^{3} G_{\alpha}}{\partial p_{k} \partial p_{m} \partial p_{n}}\left(P_{m \beta}^{i} P_{n \gamma}^{j}-P_{m \gamma}^{i} P_{n \beta}^{j}\right) \\
& +\mathrm{FL}^{*} \frac{\partial^{3} G_{\beta}}{\partial p_{k} \partial p_{m} \partial p_{n}}\left(P_{m \gamma}^{i} P_{n \alpha}^{j}-P_{m \alpha}^{i} P_{n \gamma}^{j}\right) \\
& \left.+\mathrm{FL}^{*} \frac{\partial^{3} G_{\gamma}}{\partial p_{k} \partial p_{m} \partial p_{n}}\left(P_{m \alpha}^{i} P_{n \beta}^{j}-P_{m \beta}^{i} P_{n \alpha}^{j}\right)\right]
\end{aligned}
$$

where,

$$
\begin{gathered}
P_{j \mu}^{i}=W_{j k} \mathrm{FL}^{*} \frac{\partial^{2} G_{\mu}}{\partial p_{k} \partial p_{i}} \\
P_{k \mu \nu}^{i j}=\frac{\partial W_{l m}}{\partial \dot{q}^{k}} \mathrm{FL}^{*}\left(\frac{\partial^{2} G_{\mu}}{\partial p_{i} \partial p_{l}} \frac{\partial^{2} G_{\nu}}{\partial p_{m} \partial p_{j}}-\frac{\partial^{2} G_{\nu}}{\partial p_{i} \partial p_{l}} \frac{\partial^{2} G_{\mu}}{\partial p_{m} \partial p_{j}}\right) \\
+W_{l m} W_{k n} \mathrm{FL}^{*} \frac{\partial}{\partial p_{n}}\left(\frac{\partial^{2} G_{\mu}}{\partial p_{i} \partial p_{l}} \frac{\partial^{2} G_{\nu}}{\partial p_{m} \partial p_{j}}-\frac{\partial^{2} G_{\nu}}{\partial p_{i} \partial p_{l}} \frac{\partial^{2} G_{\mu}}{\partial p_{m} \partial p_{j}}\right)
\end{gathered}
$$

The main results presented in this paper are the explicit expressions for the lagrangian gauge structure functions $E_{\mu \nu}^{i j}, D_{\alpha \beta \gamma}^{i \rho}$ and $M_{\alpha \beta \gamma}^{i j k}$ given by the equations (73), (80) and (84). These equations show how the higher-order lagrangian structure tensors are determined by the hamiltonian constraints and the hamiltonian first-order structure functions. To determine these lagrangian structure tensors no knowledge of the higher-order hamiltonian structure functions is required. Notice that for the lagrangian gauge algebra to be open 
$\left(E_{\mu \nu}^{i j} \neq 0\right)$ it is necessary to have at least two constraints that depend nonlinearly on the

momenta. In order to have nonvanishing third-order structure functions $D_{\alpha \beta \gamma}^{i \rho}$, the first-order structure functions $C_{\mu \nu}^{\eta}$ must depend on the canonical momenta $p_{i}$. The third derivatives of the constraints with respect to the canonical momenta determine the fourth-order structure tensors $M_{\alpha \beta \gamma}^{i j k}$. The method presented here can be used to obtain tensors of even higher orders.

I would like to thank Gordon Semenoff for helpful discussions and comments.

\section{References}

[1] P.A.M. Dirac, Proc. R. Soc. Lond. A246, 333 (1958).

[2] E.S. Fradkin, G.A. Vilkovisky, Phys. Lett. B55, 224 (1975); CERN Report Th-2332 (1977).

[3] D.Z. Freedman, P. van Nieuwenhuizen, Phys. Rev. 14D, 912 (1976).

[4] R.E. Kallosh, Nucl. Phys. B141, 141 (1978).

[5] B. de Wit, J.W. van Holten, Phys. Lett. B79, 389 (1978).

[6] I.A. Batalin and G.A. Vilkovisky, J. Math. Phys. 26, 172 (1985).

[7] E.C.G. Sudarshan, N. Mukunda, "Classical Dynamics: A Modern Perspective", Wiley, New York (1974).

[8] D.M. Gitman, V.I. Tyutin, "Quantization of Fields with Constraints", Springer, Berlin (1990)

[9] I.A. Batalin and G.A. Vilkovisky, Phys. Lett. B102, 27 (1981).

[10] J. Gomis, J. Paris, S. Samuel, Phys. Rep. 259, 1 (1995).

[11] M. Chaichian, D. Louis-Martinez, Phys. Rev. 46D, 1799 (1992).

[12] P.A.M. Dirac, "Lectures in Quantum Mechanics", Yeshiva University, New York (1964).

[13] C. Battle, J. Gomis, J.M. Pons and N. Roman-Roy, J. Math. Phys 27, 2953 (1986).

[14] M. Henneaux, Phys. Rep. 126, 1 (1985). 